Scientific Visualization, 2021, volume 13, number 2, pages $104-116$, DOI: 10.26583/sv.13.2.08

\title{
Processing of Visual Results of a Generalized Computational Experiment for the Problem of Supersonic Flow Around a Cone at an Angle of Attack
}

\author{
A.E. Bondarev ${ }^{1}$ \\ Keldysh Institute of Applied Mathematics RAS \\ ${ }^{1}$ ORCID: 0000-0003-3681-5212, bond@keldysh.ru
}

\begin{abstract}
The work is devoted to the problems of processing and visualizing the results of a generalized computational experiment. The generalized computational experiment makes it possible to carry out parametric studies in a grid partition of the region of the space of defining parameters. The construction of a generalized computational experiment is based on the synthesis of mathematical modeling, parallel technologies and visual analytics. As a rule, the results obtained in such an experiment are multidimensional data, on the basis of which the objective functional is constructed, which is the main object of interest of the researcher. The desired end goal is to represent the dependence of the required functional on the defining parameters in an analytical form. The results of a generalized computational experiment on a comparative assessment of the accuracy of different solvers based on the problem of supersonic flow around a cone at an angle of attack are considered as an example. The results for the objective functional are presented in visual form and in the form of a group of secondorder polynomials.
\end{abstract}

Keywords: parametric studies, generalized computational experiment, processing of visual results, analytical form.

\section{Introduction}

In the 5os of the 2oth century, experimental research was the main source of information in gas dynamics, since the possibility of numerical modeling did not exist at that time. To carry out parametric studies, a long series of physical experiments had to be carried out. To obtain the necessary information, a huge number of experiments were carried out, and most of them were spent on finding out which of the key parameters did not affect the flow under study, as noted in [1]. Nevertheless, these gigantic efforts led to the emergence of truly great generalizing formulas, which then had a decisive influence on the study of flows for decades $[1,2]$.

Now such studies can be carried out on the basis of solving problems of mathematical modeling on modern high-performance computing technology. A useful tool for efficiently carrying out such calculations can be the construction of a generalized computational experiment. A generalized computational experiment is a computational technology for carrying out parametric studies in the space of the defining parameters of the problem under study, specified by the ranges of these parameters. This approach is a synthesis of solving problems of mathematical modeling based on parallel technologies and the use of visual analytics tools for data processing and visualization. In the region of the space of defining parameters, a grid partition is carried out. At each point of the grid, the problem of mathematical modeling is solved based on parallel technologies in a multitasking mode. The result is multidimensional data volumes that require the use of visualization and visual analytics tools to process them. The main approaches to scientific visualization in computational gas dynamics are described in $[3,4]$. Note that the general goal of research remains the same as it was many years ago - to 
obtain expressions for valuable functionals in an analytical form, that is, in the form of formulas.

It should be noted that parametric numerical studies in computational gas dynamics are currently being carried out very actively [5-8].

This paper gives an example of plotting dependences for the results of a generalized computational experiment in the problem of comparative estimation of the accuracy of several OpenFOAM solvers for a supersonic flow around a cone at an angle of attack. The parameter space is formed by three defining parameters - the Mach number, the cone half-angle and the angle of attack. Each of these parameters varies within a certain range. The problem at each point of the partition of the region of the space of the determining parameters is solved using several solvers of the open software package OpenFOAM [20]. The calculation results represent a multidimensional solution in discrete form. This solution is used to construct a valuable functional, which is an error in comparison with the well-known tabular solution [21], calculated in the L1 and L2 norms. Next, various visual representations of the obtained numerical data for a valuable functional are created and analytical dependences of the functional on the defining parameters are constructed.

\section{Background}

This work is a continuation of a large cycle of works on the development of a generalized computational experiment in problems of computational gas dynamics and its application to a number of practical problems.

Earlier in previous works were presented:

- basic approaches and methods of scientific visualization; [3.9]

- the basic principles and methods of generalized computational experiment constructing for problems of computational gas dynamics; [10-15]

- the main tasks of data visualization in a generalized computational experiment [16];

- a description of the construction and implementation of a generalized computational experiment for the problem of flow around cones at an angle of attack for various solvers. The problem was considered as a 4-dimensional one, where the Mach number, the cone halfangle, the angle of attack, and the choice of the solver were considered as 4 variable parameters[17-19].

\section{Visual representation of target functionals as a function of several variables}

In modern computational gas dynamics, today it is realistic to carry out a generalized computational experiment with no more than 6 defining parameters. And this is a rather unique case. Splitting into 10 points for each of the 6 parameters entails the need to solve $10^{6}$ problems. Usually, problems are considered that have 4 or 5 defining parameters.

The main method for processing data of this dimension is dimensionality reduction, which is carried out using variance analysis or transition to the space of the first principal components. This makes it possible to use well-developed methods of scientific visualization considering target functionals as a function of many variables.

Visualization is needed, first of all, in order to get a primary idea of the type of function. Visualization paves the way for constructing the target functional dependence on the defining parameters of a multidimensional problem in an analytical form. It is the construction of analytical dependencies that is the desired end goal when implementing a generalized computational experiment.

Let's consider various ways of visual representation of a function of many variables from the point of view of the subsequent analytical presentation. First, consider the function of 2 variables $\mathrm{F}(\mathrm{X}, \mathrm{Y})$. Such a function can be quite simply represented in three-dimensional form as a surface that depends on two variables (Fig. 1). Function values can be represented by contour coloring. 


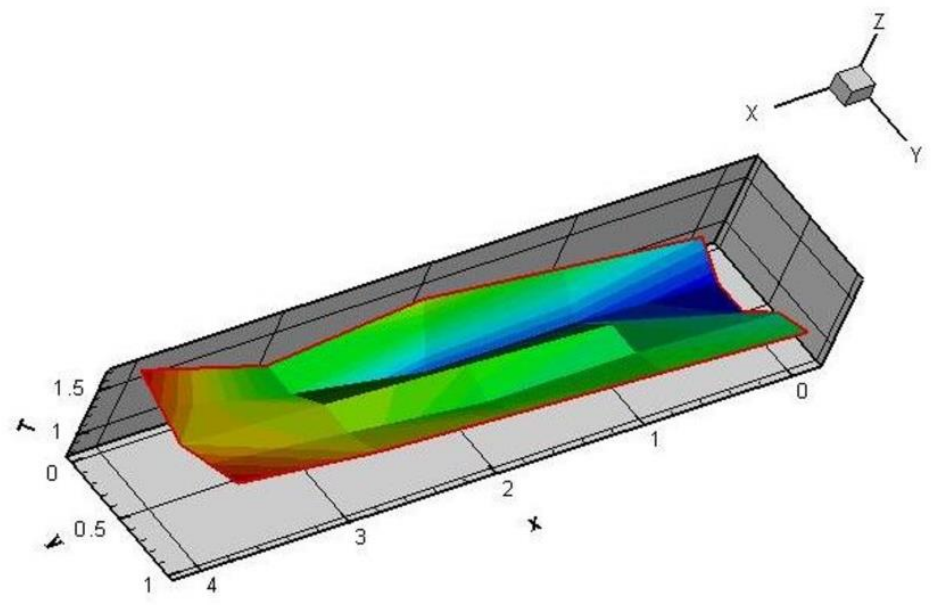

Fig 1. An example of a visual representation for function of two variables as $3 \mathrm{D}$ surface

Another way of representing is a surface unfolding on a plane of two variables with coloring along contours (Fig. 2).

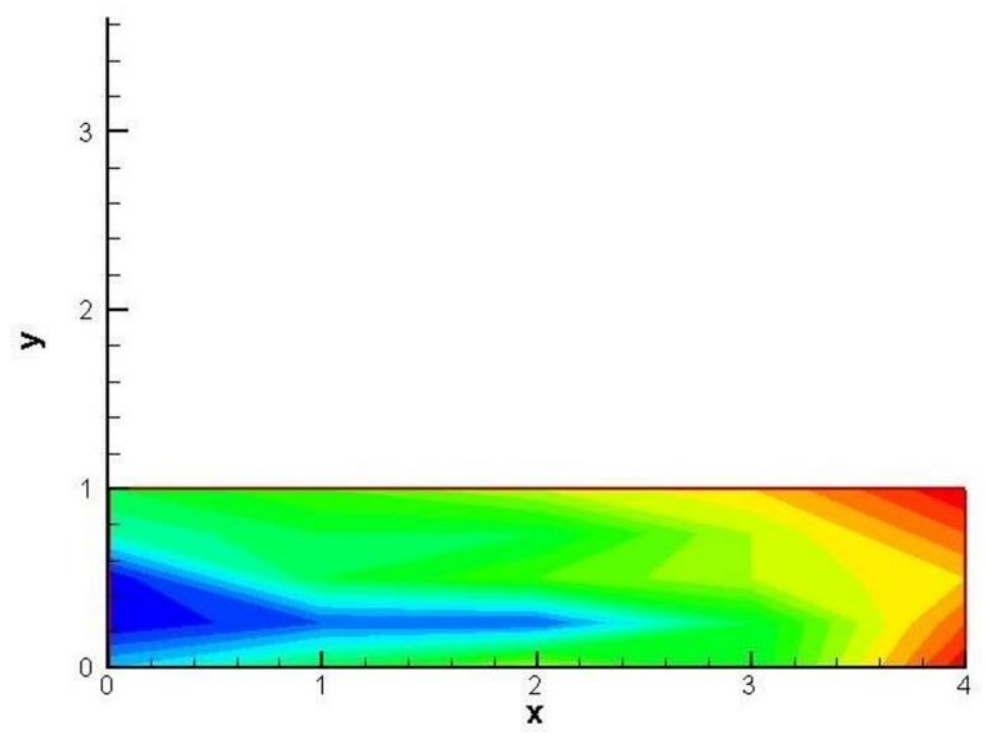

Fig 2. An example of a visual representation of a function of two variables in the form of a coloring of isolines

Note that such a representation allows one to obtain information about the shape of the surface and construct its approximation in an analytical form. For example, in the form of a plane or in the form of a second order polynomial.

For the case of three variables F (X, Y, Z), there are a number of generally accepted options for visual representation, which, as a rule, are implemented as functional modules in software packages for data visualization. For example, in the form of parallel sections (Fig. 3) or cross-sections (Fig. 4). 


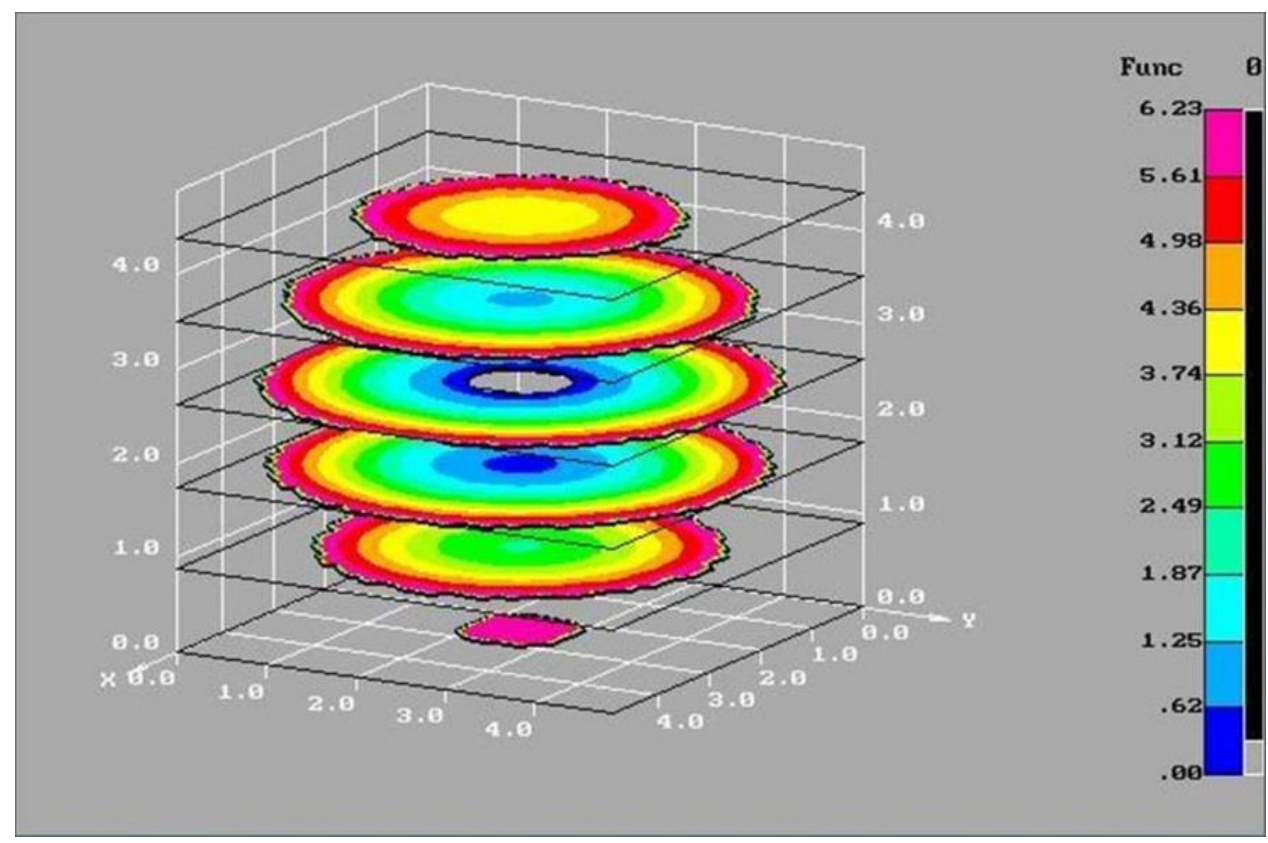

Fig 3. An example of parallel sections

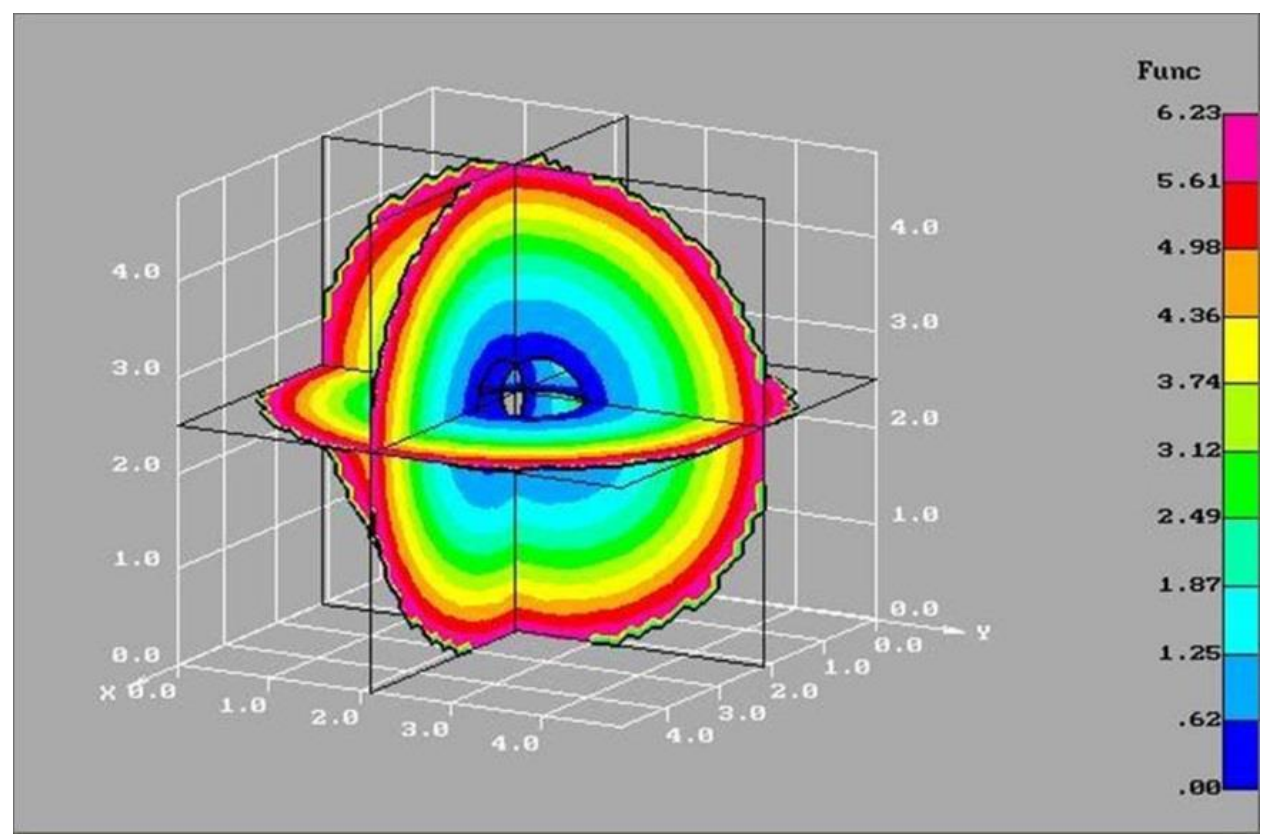

Fig 4. An example of cross-sections

Such representations allow one to obtain some information about the behavior of a function of three variables and, of course, do not exclude the possibility of approximating the function in one way or another. However, when constructing an approximation, it is often quite difficult to correlate the appearance of a function and an analytical dependence. The data presented in Figures 3 and 4 are not typical cases as they represent the radius of a sphere.

Let us consider the application of these visualization methods in order to construct an analytical dependence for the results of a specific generalized computational experiment for the comparative assessment of the accuracy of OpenFOAM solvers on the problem of supersonic flow around a cone at an angle of attack. 


\section{Analysis of the results of a comparative assessment of the accuracy of several solvers for the problem of flow around a cone in a parametric setting}

In the generalized computational experiment, a supersonic flow around a cone at an angle of attack is simulated. The Mach number, the cone half-angle and the angle of attack vary within certain ranges. The solution is carried out using three different solvers of the OpenFOAM open software package [20]. Three solvers were chosen for the solution - rhoCentralFOAM ( $\mathrm{rCF}$ ), pisoCentralFOAM (pCF), and sonicFOAM. For each fixed triplet of values of the defining parameters within the ranges of variation (Mach number, cone half-angle and angle of attack), a comparison is made with the well-known tabular solution [21] according to the L1 and L2 norms. The problem statement, solution and results are described in detail in [1719]. Thus, we get a function of the dependence of the error on 4 variables - three defining parameters and the choice of a solver. Recall that our main goal is to present the obtained numerical solution in an analytical form.

First, consider the dependence of the error for the rCF solver at zero angle of attack. In this case, we consider a function of two variables - the Mach number and the half-angle of the cone. The error surface is shown in Figure 5.

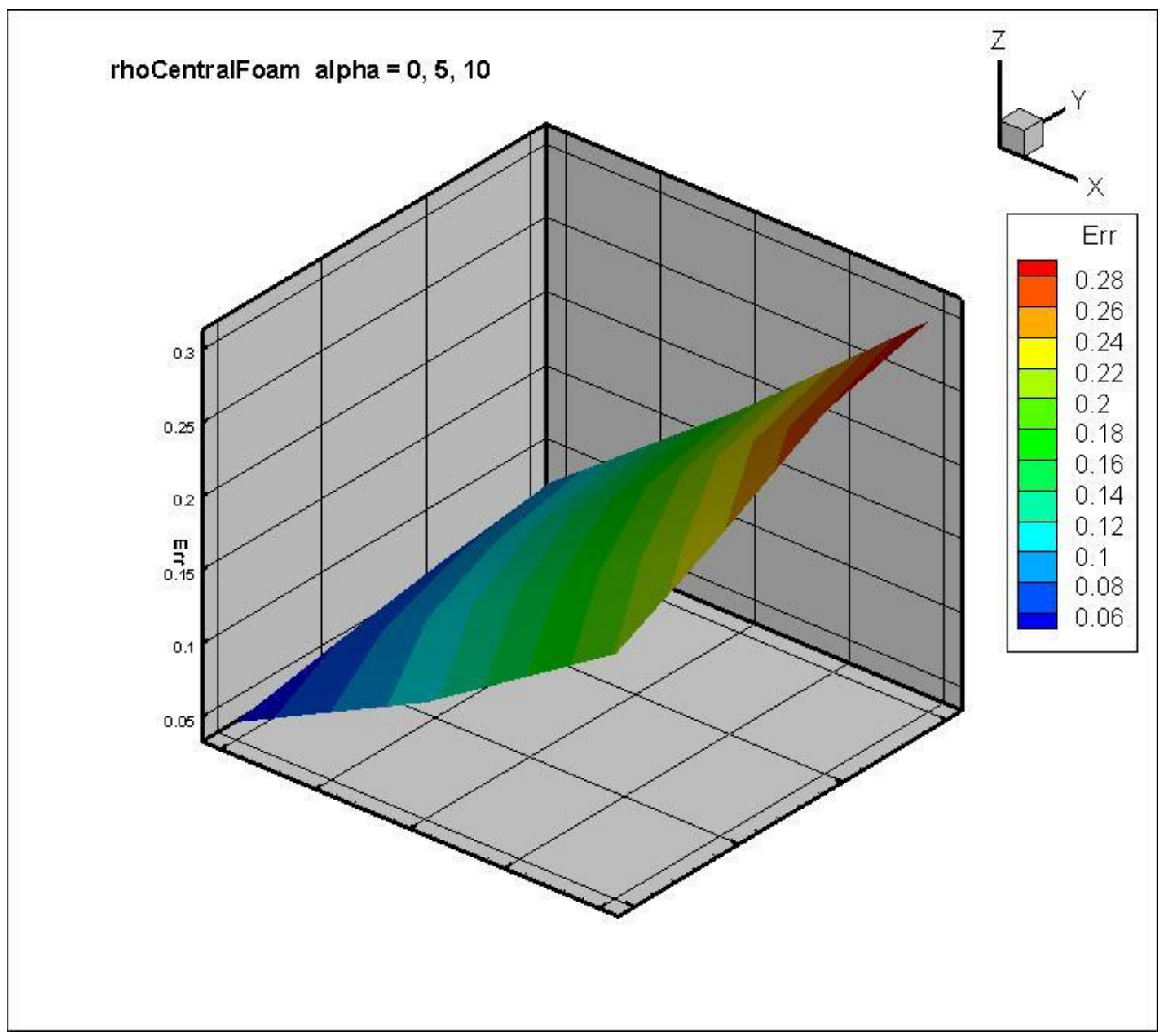

Figure 5. Error surface at $\alpha=0^{\circ}$ for the rhoCentralFoam solver

We can try to approximate this surface in two ways - either by a plane or by a surface of the second order. form

Plane approximation gives the following results. The plane equation is written in general

$$
\mathrm{AX}+\mathrm{BY}+\mathrm{CF}_{\text {err }}+\mathrm{D}=\mathrm{O}
$$


Here $\mathrm{X}$ is the Mach number $\mathrm{M}, \mathrm{Y}$ is the half-angle $\beta$ of the cone, $\mathrm{F}_{\text {err }}$ is the error of comparison with the exact solution in the L2 norm. Coefficients A, B, C, D are calculated for a specific surface. Figure 5 shows that although the surface is close to a plane, it still differs from it. To obtain a more accurate approximation, we build a plane using several different triples of surface points, and then we average the coefficients. As a result, we get

$$
\begin{aligned}
& A=0.292 \\
& B=0.0277 \\
& C=-6.2224 \\
& D=1
\end{aligned}
$$

This formula can already be used to interpolate the error, but only for the given solver $\mathrm{rCF}$ and only for the angle of attack $\alpha=0^{\circ}$. However, further constructions of such surfaces with variation of the angle $\alpha$ and with a different choice of the solver showed that the difference between the surface and the plane sharply increases, which will be seen in the subsequent figures. Consequently, plane approximation in the general case of data analysis does not suit us. To approximate curved surfaces, we use second-order polynomials, where the error for the surface under consideration can be represented as a function of the following form:

$$
\mathrm{F}_{\mathrm{err}}=\mathrm{AX}+\mathrm{BY}+\mathrm{CX}^{2}+\mathrm{DY}^{2}+\mathrm{EXY}+\mathrm{F}
$$

Here also $\mathrm{X}$ is the Mach number $\mathrm{M}, \mathrm{Y}$ is the half-angle $\beta$ of the cone, $\mathrm{F}_{\text {err }}$ is the error of comparison with the exact solution in the L2 norm. Coefficients A, B, C, D. E, F are calculated for a specific surface.

Approximating the required surface by a polynomial of the form (2) by the least squares method, we obtain

$$
\begin{aligned}
& A=0.00852295 \\
& B=0.00564067 \\
& C=0.0013973125 \\
& D=0.00014362 \\
& E=0.00110543 \\
& F=-0.0967782625
\end{aligned}
$$

The constructed surface of the second order gives the following deviation from the numerical solution

$$
\begin{aligned}
& \text { Errmin }=0.000174 \\
& \text { Errmax }=0.00482
\end{aligned}
$$

Errmean $=0.00232$

Here Errmin is the minimum deviation from the numerical solution, Errmax is the maximum deviation, Errmean is the mean deviation. Thus, the obtained approximation by a second-order polynomial coincides well with the original surface and can serve as the required analytical expression.

However, this is a solution for a function of two variables $\mathrm{Z}(\mathrm{X}, \mathrm{Y})$ with a zero angle of attack $\alpha=0^{\circ}$ and a fixed choice of the solver rCF. Let us now consider the results for a fixed choice of the same solver rCF, but in the form of a function of three variables $\mathrm{F}_{\text {err }}(\mathrm{X}, \mathrm{Y}, \mathrm{Z})$, where $\mathrm{X}$ is the Mach number $\mathrm{M}, \mathrm{Y}$ is the half-angle of the cone $\beta, \mathrm{Z}$ is the angle of attack $\alpha$, $\mathrm{F}_{\text {err }}$ is the comparison error with an exact solution in the L2-norm.

Figures 6, 7 and 8 show the error function depending on three variables in the form of external boundaries of the volume (Fig. 6), parallel sections (Fig. 7) and cross-sections (Fig. 8). Surface coloring corresponds to contours. 


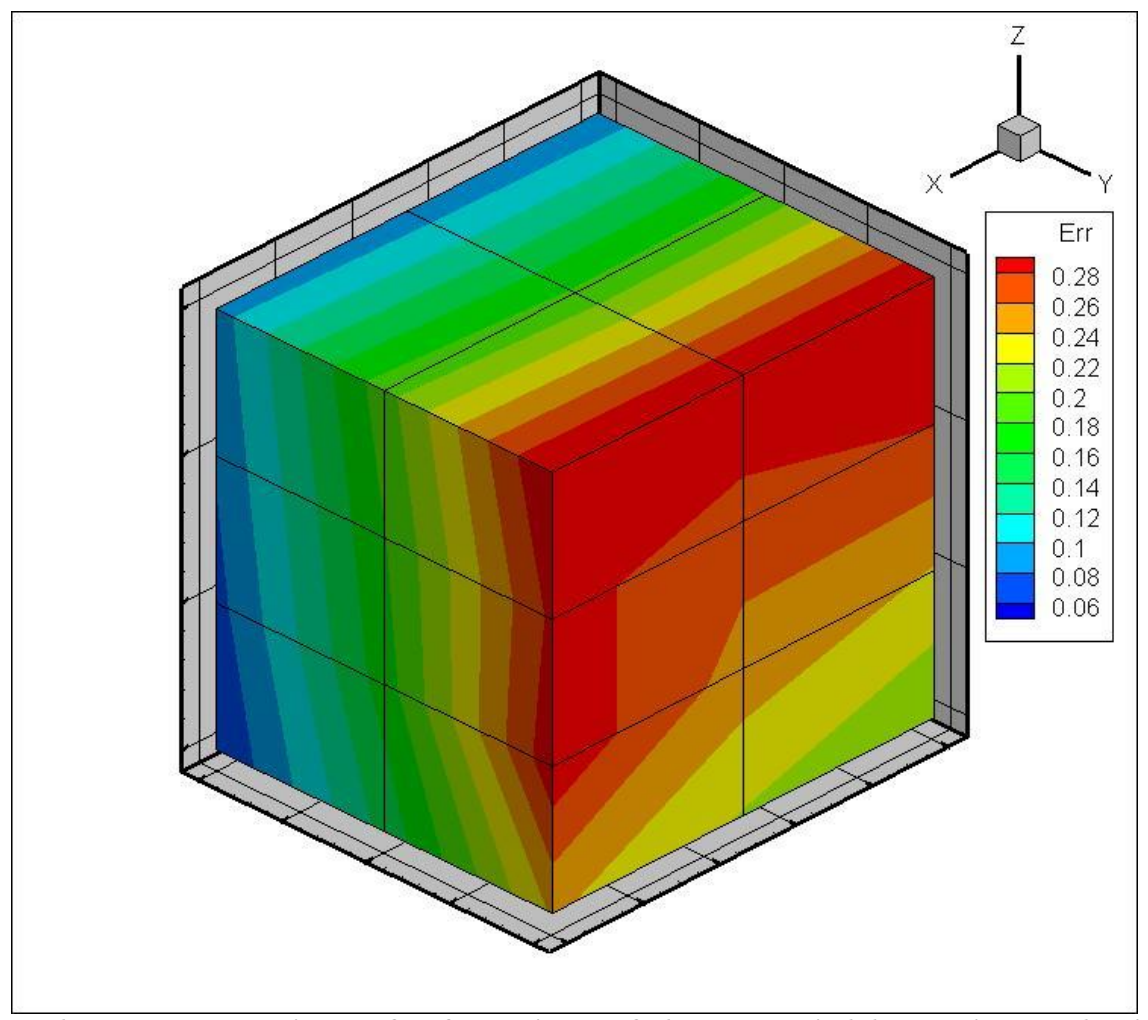

Figure 6. Visual representation of a function of three variables using coloring of external surfaces for the rhoCentralFoam solver

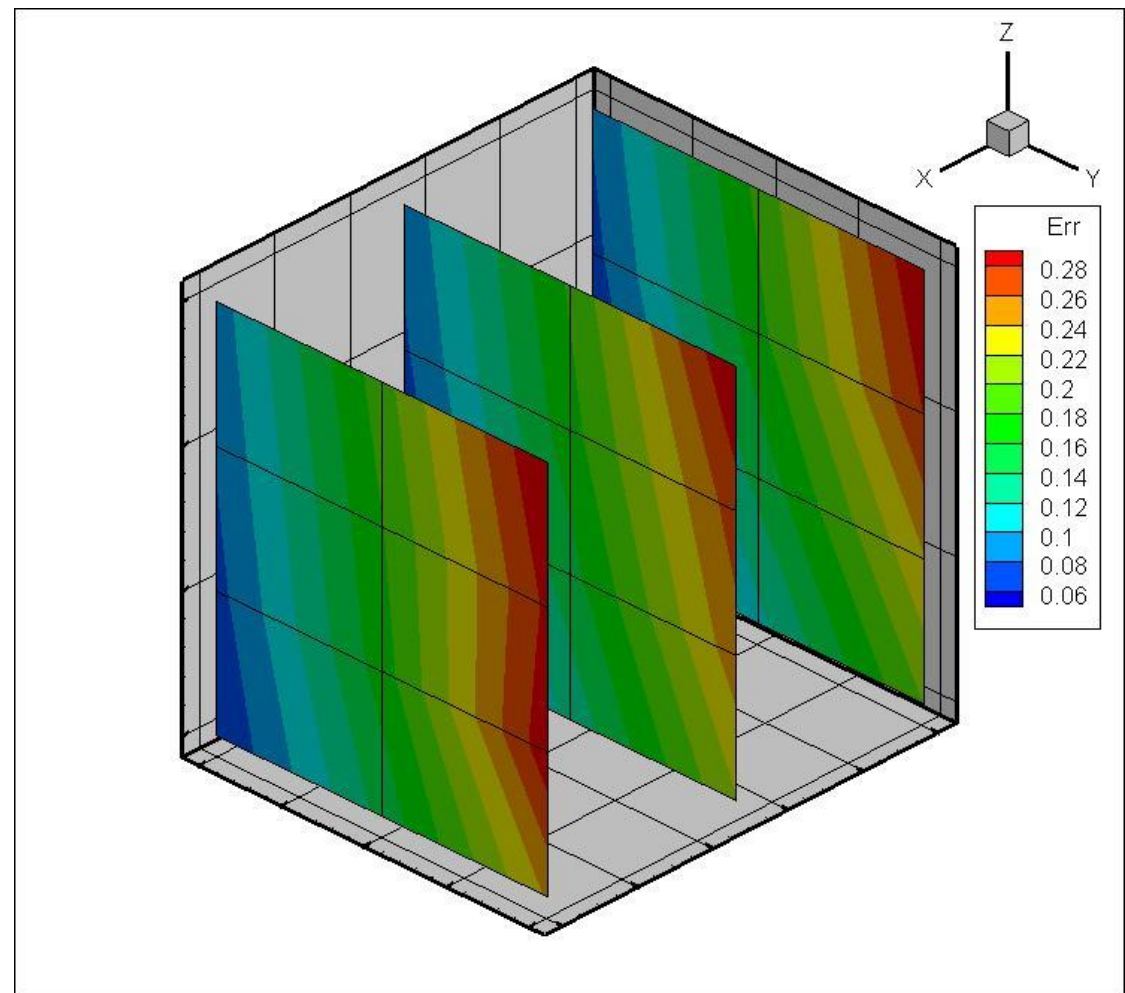

Figure 7. Visual representation of a function of three variables by parallel sections for the rhoCentralFoam solver 


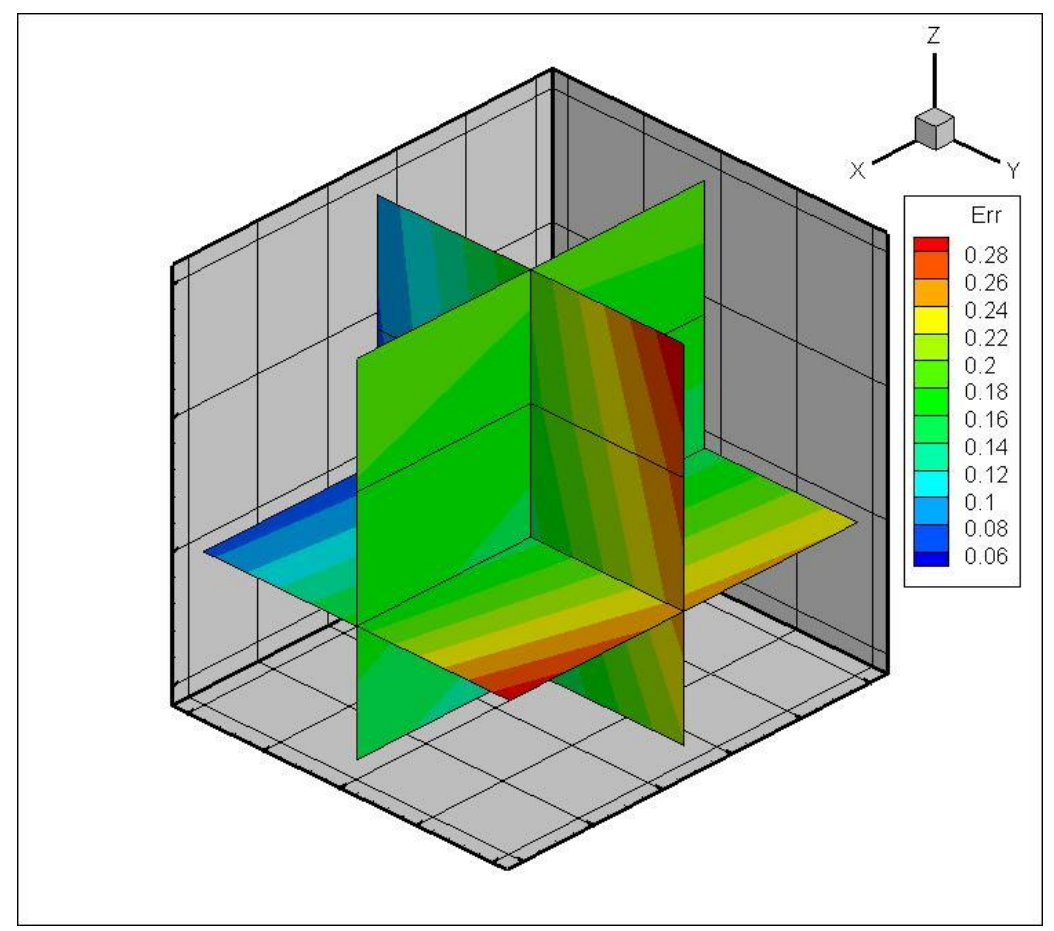

Figure 8. Visual representation of a function of three variables by cross-sections for the rhoCentralFoam solver

Figures $6,7,8$ give us some idea of the behavior of a function of three variables, but in terms of hints about how to approximate this function, these figures are clearly not informative enough.

Let's use another way. Let us analyze the initial data from the point of view of the variance of the considered function in all directions. The smallest scatter is observed for the $\mathrm{Z}$ direction, i.e. for angle $\alpha$.

Let us represent in Figure 9 the sought function in the form of several surfaces of the dependence of the error on two variables X and $\mathrm{Y}$ (the Mach number and the angle of the halfopening of the cone $\beta$ ). Each surface is constructed for its value $\alpha=0^{\circ}, 5^{\circ}, 10^{\circ}$.

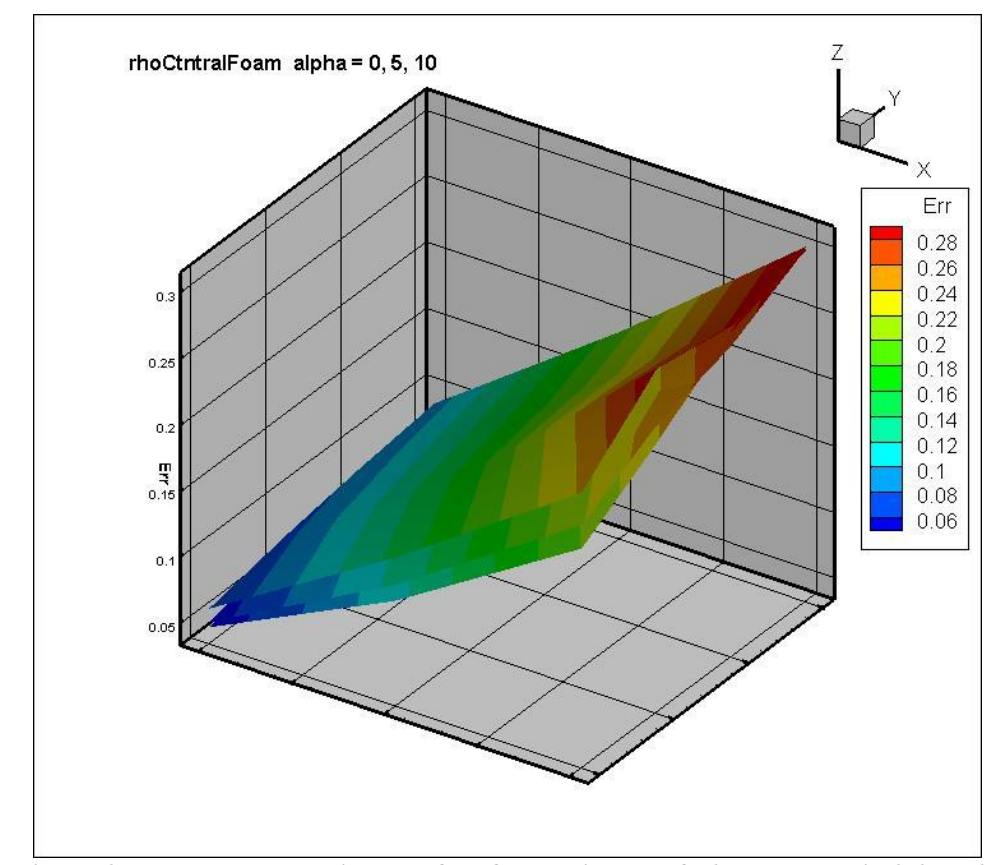

Figure 9. Visual representation of a function of three variables by 3 surfaces for the rhoCentralFoam solver 
Such a representation allows one to construct a general analytical representation of the solution in the form of three formulas of the form (2). The coefficients of the formulas are presented in Table 1 for the values $\alpha=0^{\circ}, 5^{\circ}, 10^{\circ}$.

Table 1. Coefficients for the rCF solver

\begin{tabular}{|l|l|l|l|}
\hline & $\alpha=\mathrm{O}^{\circ}$. & $\alpha=5^{\circ}$. & $\alpha=10^{\circ}$. \\
\hline A & 0.00852295 & 0.0139543625 & 0.0247155125 \\
\hline B & 0.00564067 & 0.003608695 & 0.008172105 \\
\hline C & 0.0013973125 & 0.00168559375 & 0.0018172825 \\
\hline E & -0.00014362 & -0.00007796 & -0.000162213 \\
\hline F & 0.00110543 & 0.000753045 & 0.000373555 \\
\hline
\end{tabular}

Let's construct a similar representation for pCF solver. The corresponding surfaces are shown in Figure 10.

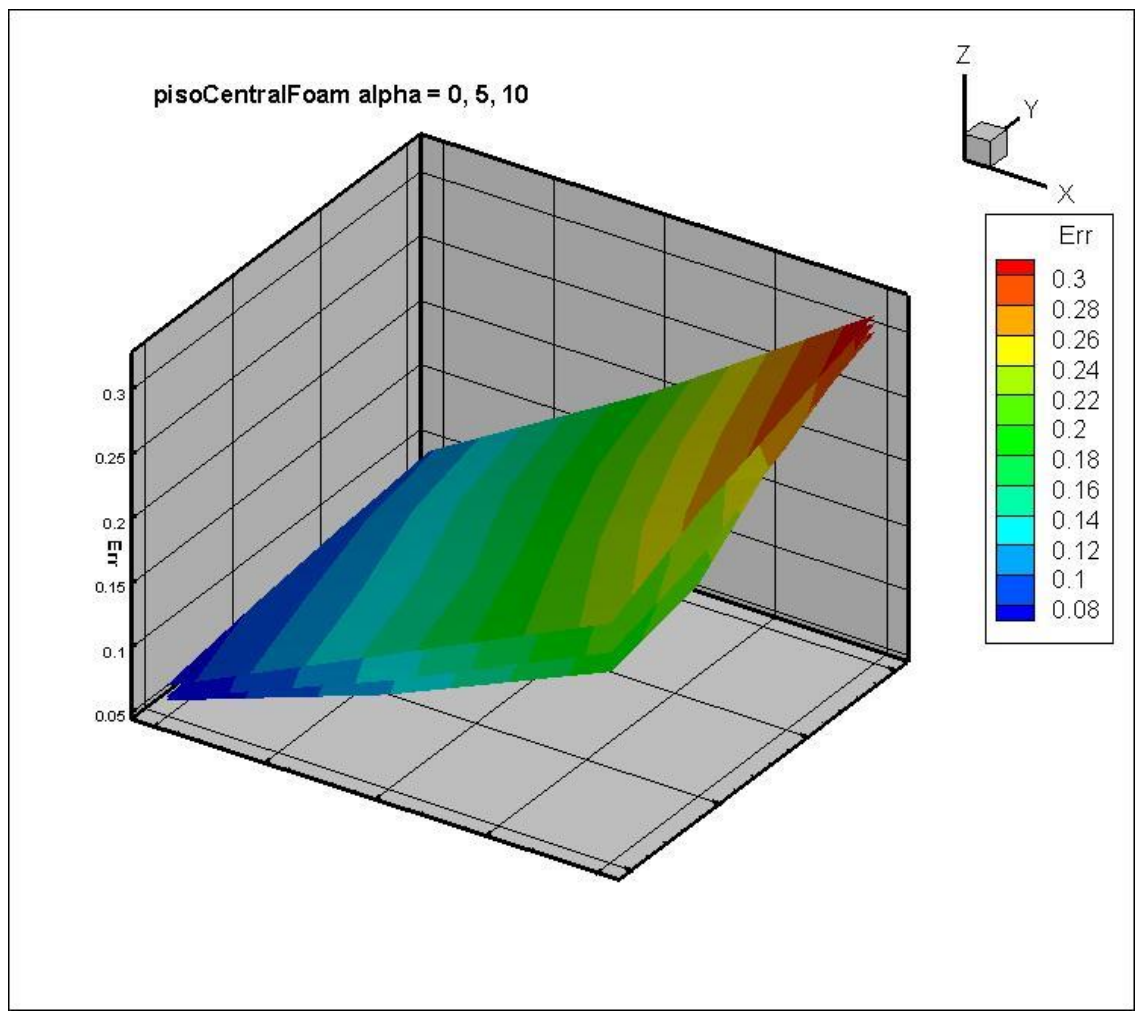

Figure 10. Visual representation of a function of three variables by 3 surfaces for the pisoCentralFoam solver.

The analytical presentation of the results for this solver is constructed in a similar way in the form of three formulas of the form (2). The corresponding coefficients are presented in table 2.

Table 2. Coefficients for the pCF solver

\begin{tabular}{|l|l|l|l|}
\hline & $\alpha=\mathrm{O}^{\circ}$. & $\alpha=5^{\circ}$. & $\alpha=10^{\circ}$. \\
\hline A & -0.003968725 & 0.0002224875 & 0.0112924375 \\
\hline B & -0.003894123 & -0.00328819167 & 0.00056873167 \\
\hline C & 0.001483375 & 0.00179471875 & 0.00158521875 \\
\hline E & 0.0000388167 & 0.000040267 & -0.00003554 \\
\hline F & 0.00141366 & 0.001141345 & 0.000927375 \\
\hline
\end{tabular}

The results for the last solver $\mathrm{sF}$ participating in the comparison are presented in Figure 11 and in Table 3. The appearance of surfaces in Figure 11 unambiguously indicates the need for their approximation by second-order surfaces. 


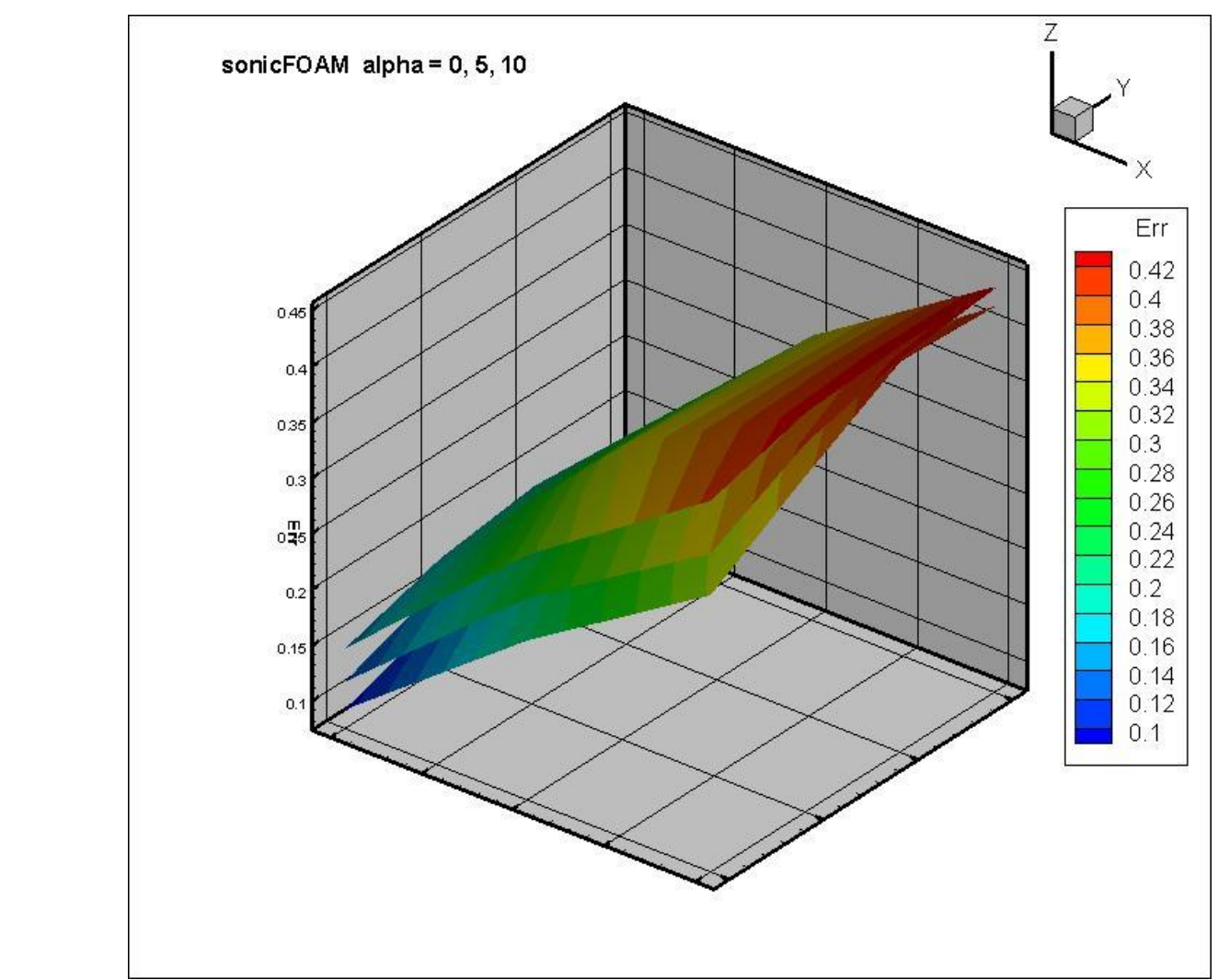

Figure 11. Visual representation of a function of three variables by 3 surfaces for the sonicFoam solver.

Table 3. Coefficients for the sF solver

\begin{tabular}{|l|l|l|l|}
\hline & $\alpha=0^{\circ}$. & $\alpha=5^{\circ}$. & $\alpha=10^{\circ}$. \\
\hline A & 0.08647875 & 0.0957112125 & 0.1270478875 \\
\hline B & 0.02144245 & 0.0172365783 & 0.0183578683 \\
\hline C & -0.0038405625 & -0.00395865625 & -0.00613859375 \\
\hline D & -0.00043633 & -0.0003262867 & -0.00035893 \\
\hline E & 0.0007405 & 0.000353035 & -0.000037645 \\
\hline F & -0.3955929375 & -0.33660227292 & -0.37349219375 \\
\hline
\end{tabular}

Thus, the coefficients in tables 1, 2, 3 for formula (2) fully provide the representation of the obtained numerical results of a generalized computational experiment in analytical form. The values at the points located between the nodes of the grid division of the region of the defining parameters can be found using interpolation.

\section{Conclusion}

The paper considers the processing and visualization of the results of a generalized computational experiment using a specific example. As an example, we used the results of a generalized computational experiment on the comparative assessment of the accuracy of three solvers of the OpenFOAM open software package. The problem of supersonic inviscid flow around a cone at an angle of attack is used as the basic problem. The space of the defining parameters is set by varying three parameters in the selected ranges - the Mach number, the cone half-angle and the angle of attack. For each solver, a discrete solution is obtained in the form of a dependence of the error on the governing parameters. A visual representation of the solution is shown, analytical forms of the solution are given as a group of 2nd order polynomials. 


\section{Acknowledgements}

The study was supported by a grant from the Russian Science Foundation № 18-1100215, https://rscf.ru/project/18-11-00215/

\section{References}

[1] Bondarev E.N., Dubasov V.T., Ryzhov Y.A., Svirschevsky S.B. and Semenchikov N.V. 1993 Aerigidromeckanika. (Moscow: Mashinostroenie) p 608 [In Russian]

[2] Kozlov L.V. "Experimental investigation of surface friction on a flat plate in a supersonic flow in the presence of heat transfer". 1963 Izvestiya AN SSSR, Mekhanika i mashinostroenie 2 p11-20 [In Russian]

[3] Bondarev A.E., Galaktionov V.A., Chechetkin V. M. Analysis of the Development Concepts and Methods of Visual Data Representation in Computational Physics / Computational Mathematics and Mathematical Physics, 2011, Vol. 51, No. 4, pp. 624-636.

[4] K. N. Volkov, V. N. Emelyanov, I. V. Teterina, M. S. Yakovchuk, "Methods and concepts of vortex flow visualization in the problems of computational fluid dynamics", Num. Meth. Prog., 17:1 (2016), 81-100.

[5] E. V. Myshenkov, V. I. Myshenkov, "Numerical simulation of efflux from Znamenskii's nozzle: Parametric investigations", TVT, 37:1 (1999), 142-149; High Temperature, 37:1 (1999), 136-143

[6] K. N. Volkov, V. N. Emelyanov, M. S. Yakovchuk, "Multiparameter optimization of operating control by the trust vector based on the jet injection into the supersonic part of a nozzle", Num. Meth. Prog., 19:2 (2018), 158-172.

[7] Rahim M.F.A., Jaafar A.A., Mamat R., Taha Z. (2020) Parametric Study of CNG-DI Engine Operational Parameters by Using Analytical Vehicle Model. In: Osman Zahid M., Abd. Aziz R., Yusoff A., Mat Yahya N., Abdul Aziz F., Yazid Abu M. (eds) iMEC-APCOMS 2019. iMEC-APCOMS 2019. Lecture Notes in Mechanical Engineering. Springer, Singapore. https://doi.org/10.1007/978-981-15-0950-6 93

[8] Zangeneh, Rozie (2021) Parametric Study of Separation and Reattachment in Transonic Airfoil Flows, AIAA Journal, DOI: 10.2514/1.Jo60520

[9] Bondarev A.E., Galaktionov V.A. Current Visualization Trends in CFD Problems / Applied Mathematical Sciences, Vol. 8, 2014, no. 28, PP.1357 - 1368, http://dx.doi.org/10.12988/ams.2014.4155

[10]Bondarev A.E. Analysis of Space-Time Flow Structures by Optimization and Visualization Methods // Transactions on Computational Science XIX, LNCS 7870, pp. 158-168.

[11]Bondarev A.E, Galaktionov V.A. Parametric Optimizing Analysis of Unsteady Structures and Visualization of Multidimensional Data // International Journal of Modeling, Simulation and Scientific Computing, 2013, V.04, N suppo1, 13 p., DOI 10.1142/S1793962313410043

[12]Bondarev A.E. Multidimensional data analysis in CFD problems / Scientific Visualization. V.6, № 5, p.59-66, 2014.

[13]Bondarev A.E., Galaktionov V.A. Multidimensional data analysis and visualization for time-dependent CFD problems // Programming and Computer Software, 2015, Vol. 41, No. 5, pp. 247-252, DOI:10.1134/So361768815050023

[14]Bondarev A.E. On the Construction of the Generalized Numerical Experiment in Fluid Dynamics // Mathematica Montisnigri, Vol. XLII, 2018, p. 52-64.

[15]A.E. Bondarev, V.A. Galaktionov. Generalized Computational Experiment and Visual Analysis of Multidimensional Data (2019). Scientific Visualization 11.4: 102 - 114, DOI: 10.26583/sv.11.4.09

[16]A.E. Bondarev . On visualization problems in a generalized computational experiment (2019). Scientific Visualization 11.2: 156 - 162, DOI: 10.26583/sv.11.2.12 
[17]Alexander E. Bondarev and Artem E. Kuvshinnikov. Analysis of the Accuracy of OpenFOAM Solvers for the Problem of Supersonic Flow Around a Cone // LNCS 10862, pp. 221-230, 2018. DOI:10.1007/978-3-319-93713-7_18

[18]Bondarev A., Kuvshinnikov A. Comparative Estimation of QGDFoam Solver Accuracy for Inviscid Flow Around a Cone // IEEE The Proceedings of the 2018 Ivannikov ISPRAS Open Conference (ISPRAS-2018). P. 82-87, DOI:10.1109/ISPRAS.2018.00019

[19]A.E. Bondarev, A.E. Kuvshinnikov. Analysis of the behavior of OpenFOAM solvers for 3D problem of supersonic flow around a cone at an angle of attack // CEUR Workshop Proceedings, V. 2763, 2020, p. 48-51. DOI: 10.30987/conferencearticle_5fce2771320efo. 90086903

[20]OpenFOAM Foundation. [Online]. Available: http://www.openfoam.org

[21]Babenko, K.I., Voskresenskii, G.P., Lyubimov, A.N., Rusanov, V.V.: Threedimensional ideal gas flow past smooth bodies. Nauka, Moscow (1964). (In Russian) 